\title{
Role of Bile Acids and Gut Microbiota in Parenteral Nutrition Associated Injury
}

\author{
Chandra Shekhara Manithody, Johan Van Nispen, Vidul Murali, Sonali Jain, Ashish Samaddar, Austin \\ Armstrong and Ajay Jain*
}

Department of Pediatrics, SSM Cardinal Glennon Hospital, Saint Louis University School of Medicine, USA

\begin{abstract}
Total Parenteral Nutrition (TPN) is a life-saving therapy where all nutritional requirements are provided intravenously. While this therapy is essential for individuals unable to process their nutritional needs enterically, significant complications arise such as intestinal failure associated liver injury (IFALD). IFALD includes hepatic steatosis, cholestasis, inflammation, ultimately progressing to cirrhosis and portal hypertension and some patients may need liver transplantation. The exact mechanism underlying this condition is not well understood, but studies have recently suggested that changes in gut microbiota and intraluminal bile acid signaling are known to play a role in the development of IFALD. In enterohepatic circulation with normal enteral nutrition, gut Farnesoid X Receptor (FXR) is activated by bile acids, which triggers the release of Fibroblast Growth Factor 19 (FGF19) into portal circulation. FGF19 serves to regulate intrahepatic bile acid synthesis with enteric nutrition. This signaling pathway is impaired in TPN as studies indicate decreased serum levels of FGF19 in subjects receiving TPN. Finally, gut microbiota is severely altered in TPN due to intestinal hypomobility. The shift in gut microbiota affects our immune response and promotes endotoxins that negatively affect liver function. Targeting the pathways affecting gut microbiota and bile acid signaling has promise in treating TPN associated injuries.
\end{abstract}

\section{Keywords}

TPN: Total parenteral nutrition, Bile acid, DC: Duodenal catheters, CDCA: Chenodeoxycholic acid, FGF19: Fibroblast growth factor 19, FXR: Farnesoid $\mathrm{x}$ receptor

\begin{abstract}
Abbreviations
PN: Parenteral Nutrition; JV: Jugular Vein; DC: Duodenal Catheters; USDA: United States Department of Agriculture; CDCA: Chenodeoxycholic Acid; BSEP: Bile Salt Export Pump; FGF19: Fibroblast Growth Factor 19; FXR: Farnesoid X Receptor; IFALD: Intestinal Failure Associated Liver Disease
\end{abstract}

\section{Introduction}

Parenteral Nutrition (PN) refers to the delivery of essential macro and micronutrients intravenously $[1,2]$. When $\mathrm{PN}$ meets all nutritional needs, the process is called Total Parenteral Nutrition (TPN). Since its first use in the 1960s, TPN has provided life-saving nutritional therapy for individuals who are not able to absorb nutrition for various anatomic or functional disorders, and, thus, remains a promising modality for providing nutrition in neonate, pediatric, and adult patients with lost or impaired gut activity [3].

Although the benefits of this therapy remain undisputed, enthusiasm for its use is tempered due to its association with adverse effects. One such complication that may arise is intestinal failure-associated liver injury (IFALD). Although classically acknowledged as a progressive cholestatic liver injury, IFALD can present with signs and symptoms of liver failure, with histologic evidence of steatosis, cirrhosis and portal hypertension, ultimately resulting in liver transplantation in some individuals. Patients with IFALD also experience a dis- rupted glucose and lipid metabolism [4]. Another common complication with TPN is gut atrophy, often characterized by decreased villi height, crypt depth, and enterocyte migration rates $[5,6]$.

While multiple mechanisms have been proposed, the exact etiology and pathophysiology of TPN associated injury remains elusive. Nevertheless, emerging data suggests that alterations in the gut microbiota, as well as intraluminal bile acid driven signaling, may play a critical role in both hepatic and intestinal injury. Evidence from recent

*Corresponding author: Ajay K Jain, Department of Pediatrics, SSM Cardinal Glennon Hospital, Saint Louis University School of Medicine, 1465 South Grand Blvd, St. Louis, MO 63104, USA

Accepted: February 29, 2020

Published online: March 02, 2020

Citation: Manithody CS, Nispen JV, Murali V, et al. (2020) Role of Bile Acids and Gut Microbiota in Parenteral Nutrition Associated Injury. J Hum Nutr 4(1):75-79 
studies also shows that factors occurring during TPN such as compromised intestinal permeability and inflammation, bacterial translocation, and lack of enteral nutrition are all associated with intestinal microbial dysbiosis [7], further strengthening the idea that gut microbes can influence TPN associated pathology.

\section{Role of Enteral Nutrition}

Clinical studies have shown that even small amounts of enteral nutrition can mitigate the negative effects of TPN. In a clinical study evaluating hyperbilirubinemia, a surrogate for hepatic injury with TPN, findings noted that there is reversal of cholestasis only when a majority of nutrition was given through the enteral route [8]. Several other studies have also confirmed the protective role of enteral nutrition [9].

Recent data point to complications in enterohepatic circulation (EHC) of bile acids secondary to an interruption in enteral feeding as a potential driver for TPN associated liver injury [2], underscoring the importance of luminal nutrients and luminal derived signaling in mediating TPN associated injury. Further, a lack of enteral feeding during TPN is hypothesized to disrupt hepato-protective gut originating signals via impairment of bile acid EHC. The novel idea highlighted here is that rather than the specific content of TPN solution, it is lack of luminal content as in TPN therapy which causes alterations in gut-originating signaling and contributes to liver and gut injury [2].

\section{Enterohepatic Circulation}

Normal EHC is dependent upon a rigorously regulated pattern of bile acid synthesis, conjugation, secretion and recirculation, all of which is driven by enteral nutrition. A lack of enteral nutrition during TPN impairs this homeostatic equilibrium. This calls to attention a critical question: If the gut and the liver signal, could disruption of these signaling events drive TPN associated injury?

This question has become particularly important with the discovery of the nuclear receptor Farnesoid X Receptor (FXR) and the characterization of the metabolically active protein Fibroblast Growth Factor 19 (FGF19) $[10,11]$. Bile acids, specifically chenodeoxycholic acid (CDCA), are known ligands for FXR, which is present in the gut and, maximally, in the terminal portion of the ileum [12,13]. Given the localization of FXR expression, its affinity for bile acids, and the evidence that bile acids regulate gut growth [2], it is plausible that FXR activation, or the lack thereof, may play a key role in TPN associated injury.

\section{FXR-FGF19 Axis and its Regulation by Bile Acids}

Fibroblast Growth Factor 19: During normal enterohepatic circulation with enteral nutrition, bile acids activate gut FXR. In fact, data from both animal studies and cell culture experiments have shown that there is robust activation of FXR in intestinal cells with bile acid introduction [13]. Bile acid activation of FXR in the gut results in the release of the protein Fibroblast Growth Factor 19 (FGF19), which reaches the liver via the portal system to exert metabolic effects [14].
Bile acids and Hepatic Homeostasis: When regular nutrition is being provided, FGF19 regulates intrahepatic bile acid synthesis, which is impaired with TPN therapy via FGF19 signaling suppression of intrahepatic cholesterol 7 alpha-hydroxylase (CYP7A1), a bile acid synthesis rate-limiting step [15].

Now, enterocytic release of FGF19 via gut FXR activation is widely believed to serve as a luminal signal to the liver to regulate intrahepatic bile acid synthesis. Such signaling is impaired with TPN therapy. In fact, with bile duct ligation, a quick and sustained reduction in serum FGF19 levels occurs, which further supports this mechanistic pathway [16].

Importantly, while gut FXR activation results in FGF19 release, this pathway is not active in the liver. This was confirmed by testing tissue-specific roles of FXR. Treatment by FXR agonists resulted in significant repression of CYP7A1 in liver FXR knockout mice, but not in gut FXR knockouts, attesting to the regulation of CYP7A1 by intestinal FXR activation, thus underscoring the importance of luminal signaling [17]. Indeed, animal studies have confirmed that serum FGF19 levels are significantly lower during TPN therapy than the robust levels observed upon enteral nutrition [2].

Additionally, to objectively quantify and assess whether levels of FGF19 were altered with enteral food deprivation and bile acids, serum FGF19 levels were evaluated in human patients treated with the gut FXR agonist, CDCA, or the bile acid sequestering agent, cholestyramine. Hepatic bile acid production was estimated by measuring the serum surrogate 7alpha-hydroxy-4-cholesterin-3-one (C4), which is a known marker for the enzymatic activity of CYP7A1. While cholestyramine resulted in an almost18-fold increase in the serum concentration of $\mathrm{C} 4$ with an accompanying $87 \%$ reduction in FGF19 levels, bile acid treatment combined with CDCA drove a $250 \%$ increase in FGF19 levels and a decrease in serum C4 by $26 \%$ [18]. Further corroborating these responses, animal studies have reported significant hepatic injury and gut atrophy upon TPN infusion with improvement upon delivery of enteral bile acid analogs [2,19].

\section{Lipid and Glucose Homeostasis}

The fact that TPN therapy results in glucose and lipid abnormalities is well established. Recent evidence suggests that such alteration might be secondary to altered gut-derived signaling driven by a lack of bile acid mediated gut FXR activation in conjunction with TPN infusion. In fact, recent data provides compelling evidence that FGF19 additionally regulates glycemic and lipid metabolism. In support of this theory, urine studies have shown a significant improvement in diabetes and a reduction in triglycerides in animals receiving an infusion of recombinant FGF19. FGF19 infusion also resulted in an acute decrease in messaging for the leptin receptor and acetyl coenzyme A carboxylase, both of which are key regulators of lipid homeostasis. Large animal studies have also shown that TPN induced insulin resistance and hypertriglyceridemia can be significantly improved with enteral bile acid treatment [2].

\section{Gut Microbiota and TPN}

There has been significant recent interest in critically eval- 
uating the role of gut microbiota as a driver for TPN associated injury. Due to a lack of luminal nutrition, significant alterations in the gut microbiota, as well as gut signaling, have been shown in animals undergoing TPN therapy $[2,3,19,20]$. Indeed, a consequence of not having enteral nutrition is intestinal hypomotility which drives bacterial overgrowth and clonal shifts [21].

Several animal studies evaluating morphometric analysis have demonstrated severe gut mucosal atrophy with a significantly lower villous to crypt ratio upon TPN therapy $[5,6]$. Given this data, it has been postulated and noted in studies that TPN therapy results in increased gut permeability as well as bacterial translocation secondary to such gut injury.

Bacterial permeation, worsened by bacterial overgrowth, drives endotoxin mediated down-regulation of key hepatobiliary receptors and transporters [22]. In clinical studies, newborn babies on TPN have significantly increased susceptibility to TPN associated hepatic injury secondary to endotoxin and bacterial translocation [23].

Several studies have shown an improvement in liver injury after selective antibiotic therapy which points to a direct role of gut bacteria in mediating TPN associated injury [24-26]. Rodent studies have also reported significantly higher Interleukin-6 (IL-6) and Tumor Necrosis Factor (TNF) in animals on TPN in comparison to the control animals [27-29].

Animal studies have shown a dominance of the Firmicutes phylum with normal enteral nutrition. Conversely, TPN therapy resulted in a significant proliferation of the pro-inflammatory Bacteroidetes phylum, thus altering the normal Firmicutes to Bacteroidetes ratio. Bacteroidetes are known to promote intestinal inflammation $[30,31]$ and increase intestinal permeability [32,33], which can drive bacterial flux across the mucosa and result in cytokine-mediated hepatocellular injury [34-36]. Further highlighting the importance of bile acids and microbiota, enteral bile acid treatment in animals on TPN has been found to preserve clonal gut microbiota shifts and modulate TPN associated injury [20].

Gut microbiota also influence the host immune system. There are several microbial metabolic products such as proteins and polysaccharides that activate recognition receptors such as Toll-like receptors (TLRs) and NOD-like receptors (NODs) which stimulate the mucosal immune system. The gut microbiota in turn promote a tolerogenic state within the intestinal mucosa (stimulating lymphocytes, decreased NF-kB signaling, etc.) and initiates mechanisms to prevent unchecked bacterial overgrowth [7]. Indeed, clonal shifts in the microbiota with TPN therapy are drivers for alteration in immune responses and endotoxin mediated systemic injury.

\section{Luminal Receptors, Gut Microbiota and TPN}

Emerging data also points to modulation of the gut growth regulator TGR5 by gut microbiota in mediating TPN associated injury $[19,20,37]$. Glucagon-like peptide - 2 (GLP-2), a gut growth hormone, is less activated due to a lack of gut TGR5 activation during TPN [19].
During normal enterohepatic circulation, primary bile acids synthesized hepatically undergo de-conjugation to secondary bile acids by the gut microbiota $[38,39]$.

Preferential activation of FXR is by primary bile acids, while that of TGR5 is by secondary bile acids. Therefore, gut microbiota can shift the bile acid signaling properties through the de-conjugation of primary bile acids [40-42]."

Current evidence also shows that both FXR and TGR5 can modulate the intestinal barrier, permeability, and immune responses [43-46]. One of the key FXR modulated genes is the inducible nitric oxide synthase (iNOS) $[45,47]$, which regulates host immunity as well as antimicrobial activity. Other important FXR modulated angiogenin (ANG1)-coding genes, which are involved in the acute phase response to infection and have potent antibacterial properties, and carbonic anhydrase 12 (CAR12), a host antibacterial defense enzyme $[48,49]$. Additionally, exogenous activation of TGR5 significantly decreases pro-inflammatory cytokines, specifically interleukin-1 $\alpha$ (IL-1 $\alpha$ ), IL-1 $\beta$, IL-6, and TNF- $\alpha$ [50]. Thus, there is significant support for the idea that altered gut microbiota and bile acids during TPN play a prominent role in TPN injury.

\section{Conclusion}

Total Parenteral Nutrition remains a crucial life-supporting therapy. Despite the benefits of TPN, intestinal failure associated liver disease, a disrupted glucose and lipid metabolism, and gut atrophy remain too common. Additionally, enterohepatic circulation and the gut microbiota appear to be altered with TPN, which likely drive the associated liver and intestinal injury. Bile acids in the intestinal lumen, which are ligands for FXR, activate gut FXR to release FGF19 which via portal circulation is delivered to the liver for its metabolic effects. The FGF19-FXR pathway regulates bile acid synthesis, which is disrupted in TPN therapy.

Significant alterations in the gut microbiota have also been reported with TPN use which modulate bile acid deconjugation and in turn the activation of luminal receptors driving hepatic and gut injury. Restoration of microbiota by exogenous bile acids as well as selective enteral antibiotic therapy can mitigate TPN associated injury.

Changes in the intestinal barrier, immune responses and permeability are additional drivers of TPN associated injury.

In summary, there seems to be compelling evidence that gut microbiota and bile acids play an important role in TPN associated injury. Targeting the pathways that regulate such could be key to furthering our understanding of injury associated with TPN and in future therapeutic strategies.

\section{Author Contributions}

Jain AK, Manithody C contributed to the conception/ design of the research. All authors contributed to acquisition, analysis, or interpretation of data and drafting the manuscript and its critical evaluation. All authors agree to be fully accountable for ensuring the integrity and accuracy of the work and approved the final manuscript. 


\section{Funding}

This work was supported by the National Institute of Health (Grant Number: K08DK098623-01A1 and R03 DK121046-01). Additional funding was provided to AK Jain via the Saint Louis University, internal grant mechanism and the DeNardo Foundation.

\section{Conflicts of Interest}

A.K.J. reports honorarium from Alexion Pharmaceuticals outside/unrelated to the submitted work; the other authors have indicated they have no potential conflicts of interest to disclose.

\section{References}

1. Dudrick SJ (2003) Early developments and clinical applications of total parenteral nutrition. JPEN J Parenter Enteral Nutr 27: 291299.

2. Jain AK, Stoll B, Burrin DG, et al. (2012) Enteral bile acid treatment improves parenteral nutrition-related liver disease and intestinal mucosal atrophy in neonatal pigs. American Journal of Physiology Gastrointestinal and Liver Physiology 302: 218-224.

3. Jain AK, Wen JX, Arora S, et al. (2015) Validating hyperbilirubinemia and gut mucosal atrophy with a novel ultramobile ambulatory total parenteral nutrition piglet model. Nutr Res 35: 169174.

4. Hsieh MH, Pai W, Tseng HI, et al. (2009) Parenteral nutrition-associated cholestasis in premature babies: Risk factors and predictors. Pediatrics and Neonatology 50: 202-207.

5. Kansagra K, Stoll B, Rognerud C, et al. (2003) Total parenteral nutrition adversely affects gut barrier function in neonatal piglets. Am J Physiol Gastrointest Liver Physiol 285: 1162-1170.

6. Peterson CA, Carey HV, Hinton PL, et al. (1997) GH elevates serum IGF-I levels but does not alter mucosal atrophy in parenterally fed rats. Am J Physiol 272: 1100-1108.

7. Cahova M, Bratova M, Wohl P (2017) Parenteral nutrition-associated liver disease: The role of the gut microbiota. Nutrients 9: 987.

8. Javid PJ, Collier S, Richardson D, et al. (2005) The role of enteral nutrition in the reversal of parenteral nutrition-associated liver dysfunction in infants. J Pediatr Surg 40: 1015-1018.

9. Kelly DA (2010) Preventing parenteral nutrition liver disease. Early Hum Dev 86: 683-687.

10. Wu X, Li Y (2009) Role of FGF19 induced FGFR4 activation in the regulation of glucose homeostasis. Aging 1: 1023-1027.

11. Schaap FG (2012) Role of fibroblast growth factor 19 in the control of glucose homeostasis. Curr Opin Clin Nutr Metab Care 15: 386-391.

12. Eloranta JJ, Kullak-Ublick GA (2008) The role of FXR in disorders of bile acid homeostasis. Physiology 23: 286-295.

13. Inagaki T, Choi M, Moschetta A, et al. (2005) Fibroblast growth factor 15 functions as an enterohepatic signal to regulate bile acid homeostasis. Cell Metabolism 2: 217-225.

14. Fu L, John LM, Adams SH, et al. (2004) Fibroblast growth factor 19 increases metabolic rate and reverses dietary and leptin-deficient diabetes. Endocrinology 145: 2594-2603.

15. Goodwin B, Jones SA, Price RR, et al. (2000) A regulatory cascade of the nuclear receptors FXR, SHP-1, and LRH-1 represses bile acid biosynthesis. Molecular cell 6: 517-526.

16. Naugler WE, Tarlow BD, Fedorov LM, et al. (2015) Fibroblast growth factor signaling controls liver size in mice with humanized livers. Gastroenterology 149: 728-740.

17. Kim I, Ahn SH, Inagaki T, et al. (2007) Differential regulation of bile acid homeostasis by the farnesoid $\mathrm{X}$ receptor in liver and intestine. J Lipid Res 48: 2664-2672.

18. Lundasen T, Galman C, Angelin B, et al. (2006) Circulating intestinal fibroblast growth factor 19 has a pronounced diurnal variation and modulates hepatic bile acid synthesis in man. Journal of Internal Medicine 260: 530-536.

19. Jain AK, Wen JX, Blomenkamp KS, et al. (2015) Oleanolic acid improves gut atrophy induced by parenteral nutrition. JPEN J Parenter Enteral Nutr 40: 67-72.

20. Jain AK, Sharma A, Arora S, et al. (2017) Preserved gut microbial diversity accompanies upregulation of tgr5 and hepatobiliary transporters in bile acid-treated animals receiving parenteral nutrition. JPEN J Parenter Enteral Nutr 41: 198-207.

21. Giannelli V, Di Gregorio V, lebba V, et al. (2014) Microbiota and the gut-liver axis: Bacterial translocation, inflammation and infection in cirrhosis. World J Gastroenterol 20: 16795-16810.

22. Harris JK, El Kasmi KC, Anderson AL, et al. (2014) Specific microbiome changes in a mouse model of parenteral nutrition associated liver injury and intestinal inflammation. PLoS One 9: e110396.

23. Choi SJ, Lee KJ, Choi JS, et al. (2016) Poor prognostic factors in patients with parenteral nutrition-dependent pediatric intestinal failure. Pediatr Gastroenterol Hepatol Nutr 19: 44-53.

24. Lichtman SN, Keku J, Schwab JH, et al. (1991) Hepatic injury associated with small bowel bacterial overgrowth in rats is prevented by metronidazole and tetracycline. Gastroenterology 100: 513-519.

25. Freund HR, Muggia-Sullam M, LaFrance R, et al. (1985) A possible beneficial effect of metronidazole in reducing TPN-associated liver function derangements. J Surg Res 38: 356-363.

26. Koga H, Sakisaka S, Yoshitake M, et al. (2002) Abnormal accumulation in lipopolysaccharide in biliary epithelial cells of rats with self-filling blind loop. Int J Mol Med 9: 621-626.

27. Whiting JF, Green RM, Rosenbluth AB, et al. (1995) Tumor necrosis factor-alpha decreases hepatocyte bile salt uptake and mediates endotoxin-induced cholestasis. Hepatology 22: 1273-1278.

28. Zheng YJ, Tam YK, Coutts RT (2004) Endotoxin and cytokine released during parenteral nutrition. JPEN Journal of Parenteral and Enteral Nutrition 28: 163-168.

29. Lehmann GL, Carreras FI, Soria LR, et al. (2008) LPS induces the TNF-alpha-mediated downregulation of rat liver aquaporin-8: Role in sepsis-associated cholestasis. American Journal of Physiology Gastrointestinal and Liver Physiology 294: 567-575.

30. Wu S, Powell J, Mathioudakis N, et al. (2004) Bacteroides fragilis enterotoxin induces intestinal epithelial cell secretion of interleukin-8 through mitogen-activated protein kinases and a tyrosine kinase-regulated nuclear factor-kappaB pathway. Infect Immune 72: 5832-5839.

31. Yoon YM, Lee JY, Yoo D, et al. (2010) Bacteroides fragilis enterotoxin induces human beta-defensin-2 expression in intestinal epithelial cells via a mitogen-activated protein kinase/I kappaB kinase/NF-kappaB-dependent pathway. Infect Immun 78: 20242033.

32. Remacle AG, Shiryaev SA, Strongin AY (2014) Distinct interac- 
tions with cellular E-cadherin of the two virulent metalloproteinases encoded by a Bacteroides fragilis pathogenicity island. PloS one.

33. Wu S, Lim KC, Huang J, et al. (1998) Bacteroides fragilis enterotoxin cleaves the zonula adherens protein, E-cadherin. Proc Natl Acad Sci USA 95: 14979-14984.

34. Green RM, Beier D, Gollan JL (1996) Regulation of hepatocyte bile salt transporters by endotoxin and inflammatory cytokines in rodents. Gastroenterology 111: 193-198.

35. Alrefai WA, Gill RK (2007) Bile acid transporters: Structure, function, regulation and pathophysiological implications. Pharmaceutical research 24: 1803-1823.

36. Trauner M, Arrese M, Lee H, et al. (1998) Endotoxin downregulates rat hepatic ntcp gene expression via decreased activity of critical transcription factors. J Clin Invest 101: 2092-2100.

37. Kumar JA, Teckman JH (2015) Controversies in the mechanism of total parenteral nutrition induced pathology. Children 2: 358370.

38. Ridlon JM, Kang DJ, Hylemon PB (2006) Bile salt biotransformations by human intestinal bacteria. J Lipid Res 47: 241-259.

39. Park MY, Kim SJ, Ko EK, et al. (2016) Gut microbiota-associated bile acid deconjugation accelerates hepatic steatosis in ob/ob mice. J Appl Microbiol 121: 800-810.

40. Ramirez-Perez O, Cruz-Ramon V, Chinchilla-Lopez P, et al. (2017) The role of the gut microbiota in bile acid metabolism. Ann Hepatol 16: S15-S20.

41. Fiorucci S, Distrutti E (2015) Bile acid-activated receptors, in- testinal microbiota, and the treatment of metabolic disorders. Trends Mol Med 21: 702-714.

42. Ridlon JM, Kang DJ, Hylemon PB, et al. (2014) Bile acids and the gut microbiome. Curr Opin Gastroentero 30: 332-338.

43. Cipriani S, Mencarelli A, Chini MG, et al. (2011) The bile acid receptor GPBAR-1 (TGR5) modulates integrity of intestinal barrier and immune response to experimental colitis. PloS one.

44. Duboc H, Tache Y, Hofmann AF (2014) The bile acid TGR5 membrane receptor: From basic research to clinical application. Dig Liver Dis 46: 302-312.

45. Inagaki T, Moschetta A, Lee YK, et al. (2006) Regulation of antibacterial defense in the small intestine by the nuclear bile acid receptor. Proc Natl Acad Sci USA 103: 3920-3925.

46. Verbeke L, Farre R, Verbinnen B, et al. (2015) The FXR agonist obeticholic acid prevents gut barrier dysfunction and bacterial translocation in cholestatic rats. Am J Pathol 185: 409-419.

47. Wallace JL, Miller MJ (2000) Nitric oxide in mucosal defense: A little goes a long way. Gastroenterology 119: 512-520.

48. Hooper LV, Stappenbeck TS, Hong CV, et al. (2003) Angiogenins: A new class of microbicidal proteins involved in innate immunity. Nature Immunology 4: 269-273.

49. Biet F, Locht C, Kremer L (2001) Immunoregulatory functions of interleukin 18 and its role in defense against bacterial pathogens. Journal of Molecular Medicine 80: 147-162.

50. Keitel V, Donner M, Winandy S, et al. (2008) Expression and function of the bile acid receptor TGR5 in Kupffer cells. Biochem Biophys Res Commun 372: 78-84. 